\title{
Effects of boundary layer on wedge-induced oblique detonation structures in hydrogen-air mixtures
}

\author{
Yishen Fang ${ }^{a, b, c}$, Zijian Zhang ${ }^{a, b, *}$, Zongmin $\mathrm{Hu}^{a, b}$ \\ a School of Engineering Science, University of Chinese Academy of Sciences, Beijing 100049, China \\ ${ }^{\mathrm{b}}$ Institute of Mechanics, Chinese Academy of Sciences, Beijing 100190, China \\ c Beijing Aerospace Technology Institute, Beijing 100074, China
}

\section{H I G H L I G H T S}

- Effects of boundary layer are important in abrupt transition of ODW.

- Shock-boundary layer interaction in abrupt transition of viscous ODW.

- Significantly shorter transition length in viscous ODW.

- Different evolution process in viscous ODW.

- Great importance of considering viscosity in ODW simulations and ODE designs.

\section{A R T I C L E I N F O}

Article history:

Received 22 April 2019

Received in revised form

13 June 2019

Accepted 1 July 2019

Available online 24 July 2019

Keywords:

Oblique detonation wave

Boundary layer

Viscosity

Detonation initiation

Hydrogen-air mixture

\begin{abstract}
A B S T R A C T
Oblique detonation wave (ODW) structures are studied widely in recent years, but most of them are solved by the Euler equations without considering viscosity and then effects of boundary layer. In this study, the Navier-Stokes Equations are used to simulate the wedgeinduced ODWs in hydrogen-air mixtures, and the two types of ODW transition structures at different incident Mach number $M_{i}$ are analyzed to clarify the effects of viscosity and hence the boundary layer. Results show that the effect of boundary layer on ODW structures should be classified by the types of ODW transition patterns. As for the smooth transition pattern of ODW at high Mach numbers, the effect of boundary layer can be neglected, but for the abrupt transition pattern of ODW at low Mach numbers, the effect of boundary layer is large and it changes the ODW structure greatly. Resulting from the interaction of shock and boundary layer, a recirculation zone is formed within the viscous ODW layer at $M_{i}=7$, which leads to the phenomenon that the straight oblique shock wave evolves into two sections, with the downstream one having a larger shock angle. Additionally, the corresponding transition position moves upstream, and the initiation length becomes only one third of that in inviscid ODW. The great importance of considering viscosity in ODW simulations and future designs of combustor of oblique detonation engine has been addressed.
\end{abstract}

๑) 2019 Hydrogen Energy Publications LLC. Published by Elsevier Ltd. All rights reserved.

\footnotetext{
* Corresponding author. School of Engineering Science, University of Chinese Academy of Sciences, Beijing 100049, China.

E-mail address: zhangzijian@imech.ac.cn (Z. Zhang). 


\section{Introduction}

Oblique detonation wave (ODW) has been widely studied for many years [1,2], which can be potentially used in detonation engines to achieve hypersonic propulsion with high efficiency. Currently, there are three kinds of detonation engines, namely the pulse detonation engine (PDE) [3], rotating detonation engine (RDE) [4,5], and oblique detonation engine (ODE) [6]. The ODE is considered as the most appropriate concept for hypersonic air-breathing propulsion operating at high flight Mach numbers. However, the development of ODE is still in the theoretical and numerical stage, and the physical process of ODW still needs further study.

In early researches, ODW was usually simplified to be an oblique shock wave (OSW) combined with a post-shock heat release zone [7]. However, subsequent studies [8,9]. [10-13]. demonstrated that an ODW is composed of a non-reactive OSW, an induction zone, a set of deflagration waves and an ODW surface. As shown in Fig. 1, there are two types of transitions from OSW to ODW: one is the abrupt transition, the other is the smooth transition [14]. Teng and Jiang [15] judged the type of detonation by establishing the relationship of angles between OSW and ODW. Moreover, the surface of ODW is not always stable. Recently, there have been several research studies on the surface instability of ODW [12,16-22], illustrating and quantifying the formation of fine-scale structures on the oblique detonation surfaces.

Although the ODW structures have been studied widely, most of previous studies, such as [23,24], still assume of inviscid flow. The exclusion of viscous effects is usual in detonation researches, from normal detonations travelling in straight tubes to rotating detonations in annular chambers. However, the effects of shock-boundary layer interaction on the shock bifurcation and deflagration-to-detonation transition process have been numerically found by Gamezo et al. $[25,26]$. Additionally, the influences of interaction of ODW with incoming boundary layer on the flame structures can also be observed in $[27,28]$. As for wedge-induced ODW, the effects of the boundary layer over the wedge on the ODW structures have not attracted attentions of researchers yet. In ODEs, the boundary layer may not only disturb the structure

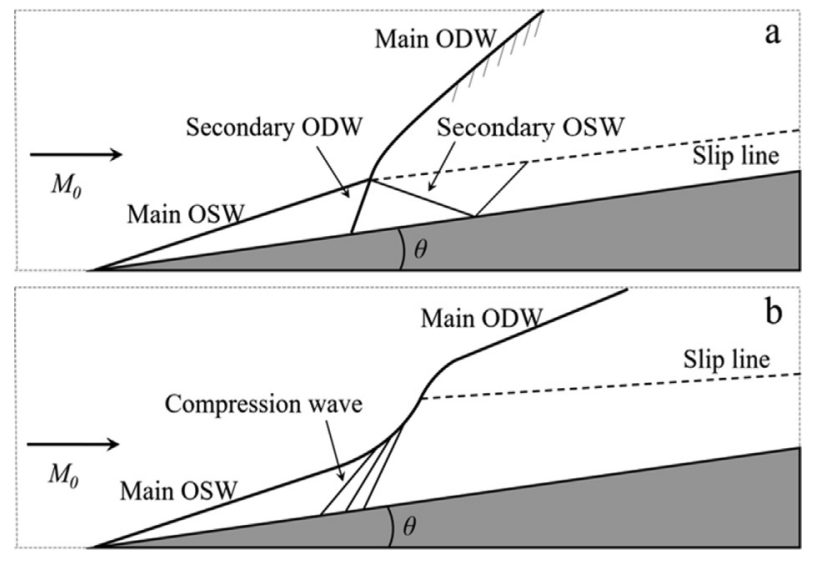

Fig. 1 - Schematic of ODWs with abrupt (a) and smooth (b) transitions. of ODW, but also change the instability of ODW. Li et al. [29] found that the viscous effect can be neglected in ODW. Thereafter, most of the studies on ODW without considering the boundary layer were based on Li's research. In general, this is reasonable because the length of the initiation zone of ODW is usually on the scale of millimeter, hence the boundary layer has not fully developed yet, resulting in a thin boundary layer. Thus, the effects of boundary layer on the whole flow field can be neglected. This is why there are so few studies on the effects of boundary layer on ODWs.

In this study, the structures of ODWs are numerically investigated using the Navier-Stokes equations, and the effects of boundary layer are discussed. It is found that the effect of boundary layer on ODW with abrupt transition structure becomes very obvious. Compared with the results of inviscid condition, the initiation structure of ODW will change greatly. The difference between viscous and inviscid increases with the strength of SOSW (secondary OSW). However, in the smooth transition structure, the role of boundary layer is consistent with the previous studies and can be neglected. In the abrupt transition structure, a recirculation zone is formed near the wall under the action of the boundary layer. This recirculation zone is adjacent to SOSW. The existence of recirculation zone changes the state of flow and chemical reactions in the initiation zone, and subsequently changes the structure of the whole initiation zone.

\section{Numerical methods}

A schematic of simulating ODWs induced by a twodimensional (2D), semi-infinite wedge is given in Fig. 2. The presence of wedge in a supersonic combustible inflow induces an OSW and a boundary layer. Notably, as for the classical configuration of an ODE [30], ODW is always induced by the OSW formed over the combustor's cowl, leading to no boundary layer existing in the incoming flow, which is also one of the assumptions made in present study. Behind the OSW, the temperature is very high. An exothermic chemical reaction begins in this zone, leading to the formation of ODW. A boundary layer develops and becomes thicker at the wall surface. The coordinate is rotated to the direction along the wedge surface and the computational domain is shown in the region enclosed by the dash lines.

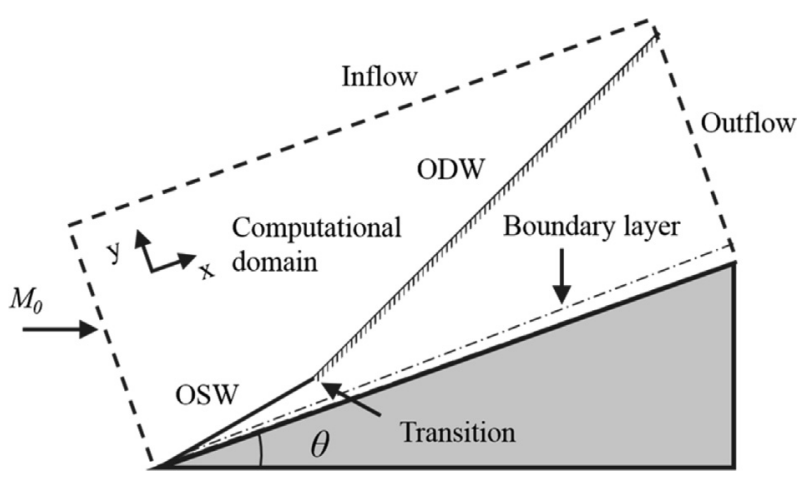

Fig. 2 - Schematic of simulating ODWs. 
The unsteady reactive Navier-Stokes equations are used as governing equations for modeling the ODW flow field, i.e.:

$\frac{\partial \tilde{U}}{\partial t}+\frac{\partial \tilde{F}}{\partial \xi}+\frac{\partial \tilde{G}}{\partial \eta}=\frac{\partial \widetilde{F_{v}}}{\partial \xi}+\frac{\partial \widetilde{G_{v}}}{\partial \eta}+\tilde{S}$

where

$\tilde{U}=\frac{U}{J}, \tilde{F}=\frac{1}{J}\left(\xi_{x} F+\xi_{y} G\right), \tilde{G}=\frac{1}{J}\left(\eta_{x} F+\eta_{y} G\right)$,

$\tilde{S}=\frac{S}{J}, \widetilde{F_{v}}=\frac{1}{J}\left(\xi_{x} F_{v}+\xi_{y} G_{v}\right), \widetilde{G_{v}}=\frac{1}{J}\left(\eta_{x} F_{v}+\eta_{y} G_{v}\right)$

with

$U=\left\{\begin{array}{c}\rho_{1} \\ \vdots \\ \rho_{n} \\ \rho u \\ \rho v \\ e\end{array}\right\}, F=\left\{\begin{array}{c}\rho_{1} u \\ \vdots \\ \rho_{n} u \\ \rho u^{2}+p \\ \rho u v \\ (e+p) u\end{array}\right\}, G=\left\{\begin{array}{c}\rho_{1} u \\ \vdots \\ \rho_{n} v \\ \rho u v \\ \rho v^{2}+p \\ (e+p) v\end{array}\right\}$,

$S=\left\{\begin{array}{c}\omega_{1} \\ \vdots \\ \omega_{n} \\ 0 \\ 0 \\ 0\end{array}\right\}, F_{v}=\left\{\begin{array}{c}0 \\ \vdots \\ 0 \\ \tau_{x x} \\ \tau_{x y} \\ u \tau_{x x}+v \tau_{x y}-q_{x}\end{array}\right\}$,

$$
G_{v}=\left\{\begin{array}{c}
0 \\
\vdots \\
0 \\
\tau_{y x} \\
\tau_{y y} \\
u \tau_{y x}+v \tau_{y y}-q_{y}
\end{array}\right\}
$$

$\frac{1}{J}=\frac{\partial(\xi, \eta)}{\partial(x, y)}=\left|\begin{array}{cc}\xi_{x} & \xi_{y} \\ \eta_{x} & \eta_{y}\end{array}\right|=\xi_{x} \eta_{y}-\xi_{y} \eta_{x}$

In the above equations, the total density and total energy are calculated by:

$\rho=\sum_{i=1}^{n} \rho_{i}, e=\rho h-p+\frac{1}{2} \rho\left(u^{2}+v^{2}\right)$

where the specific enthalpy can be written as $h=\sum_{i=1}^{n} \rho_{i} h_{i} / \rho$ with $h_{i}$ obtained from the thermodynamic data of each individual species. The equation of the state is

$p=\sum_{i=1}^{n} \rho_{i} \frac{R}{w_{i}} \mathrm{~T}$

where $w_{i}$ is the molecular weight; $\mathrm{T}$ is the gas temperature; and $\omega_{i}$ is the species' specific mass production rate determined by the chemical reaction model. The shear stresses are calculated by:

$$
\left[\begin{array}{cc}
\tau_{x x} & \tau_{x y} \\
\tau_{y x} & \tau_{y y}
\end{array}\right]=\mu\left[\begin{array}{cc}
\frac{4}{3} \frac{\partial u}{\partial x}-\frac{2}{3} \frac{\partial v}{\partial y} & \frac{\partial u}{\partial y}+\frac{\partial v}{\partial x} \\
\frac{\partial u}{\partial y}+\frac{\partial v}{\partial x} & \frac{4}{3} \frac{\partial v}{\partial y}-\frac{2}{3} \frac{\partial u}{\partial x}
\end{array}\right]
$$

For laminar flow, the viscous coefficient varies only with temperature, which is given by the Sutherland's formula [31]:

$\mu=1.458 \times 10^{-6} \frac{\mathrm{T}^{3 / 2}}{\mathrm{~T}+111} \mathrm{~N} \cdot \mathrm{s} / \mathrm{m}^{2}$

$q_{x}$ and $q_{y}$ represent the components of heat flux in two coordinate directions, respectively.

$q_{x}=k \frac{\partial T}{\partial x}, q_{x}=k \frac{\partial T}{\partial y}$

with

$k=\frac{\mu C_{p}}{\operatorname{Pr}}$

where $C_{p}$ is the constant-pressure specific heat, and $\operatorname{Pr}=0.72$ is the Prandtl number.

The governing equations are discretized on Cartesian grids and solved using the AUSMPW + finite difference scheme [32]. The latest $\mathrm{H}_{2} / \mathrm{O}_{2}$ kinetic model for high-pressure combustion [33-35], is used here, and it involves 27 reversible elementary reactions among the 8 species $\left(\mathrm{H}_{2}, \mathrm{O}_{2}, \mathrm{H}_{2} \mathrm{O}, \mathrm{H}, \mathrm{O}, \mathrm{OH}, \mathrm{HO}_{2}\right.$, and $\mathrm{H}_{2} \mathrm{O}_{2}$ ) along with the nonreactive species $\mathrm{N}_{2}$. The thermodynamic properties of the chemical species are evaluated from the 9-coefficient NASA polynomial representation [36]. A stoichiometric hydrogen-air mixture with mole ratios of $\mathrm{H}_{2}$ : $\mathrm{O}_{2}: \mathrm{N}_{2}=2: 1: 3.76$ is used. The reflecting no slip boundary is used on the wedge surface and the wedge angle is set as $25^{\circ}$. The left boundary and up boundary are set as the inflow boundary. The default inflow parameters are 1.0 atm and $300 \mathrm{~K}$. The right boundary is interpolated under the assumption of equal first-order derivatives for all flow parameters. The wall mesh is densified and the size in the y-direction is $0.005 \mathrm{~mm}$. The size is increased outward with an equal ratio of 1.05 until the same scale as the outer mesh is reached.

\section{Results and discussion}

First of all, two basic ODW structures are shown in Figs. 3 and 4 with $M_{i}=10$ and 7 , respectively, both by pressure and temperature contours. ODWs in Figs. $3 \mathrm{a}$ and $4 \mathrm{a}$ are simulated without considering the viscous effects, so they illustrate the wave structures similar to previous studies [14]. In the case of $M_{i}=10$, a smooth transition appears at around $x=0.002 \mathrm{~m}$, as shown in Fig. 3a. In contrast, an abrupt transition appears at around $x=0.015 \mathrm{~m}$ in the case of $M_{i}=7$, as shown in Fig. $4 \mathrm{a}$. It is observed that the angles of oblique shock and oblique detonation in Fig. $4 \mathrm{a}$ are larger than those in Fig. 3a, and a complicated wave system appears in the OSW-ODW transition region. The main difference of these two structures in the transition region is the formation of oblique shock/detonation surface. There are two OSWs connected with the transition point in Fig. 4a: one extending upstream and generating the SODW (secondary ODW), and the other denoted by SOSW extending downstream and reflecting on the wedge.

If the viscous terms are included in simulations, the structures become different but the effects of viscosity and hence the formed boundary layer depend on the types of ODW transition patterns. In the case of $M_{i}=10$, as shown in Fig. 3b, there is only slight difference. Due to the existence of 

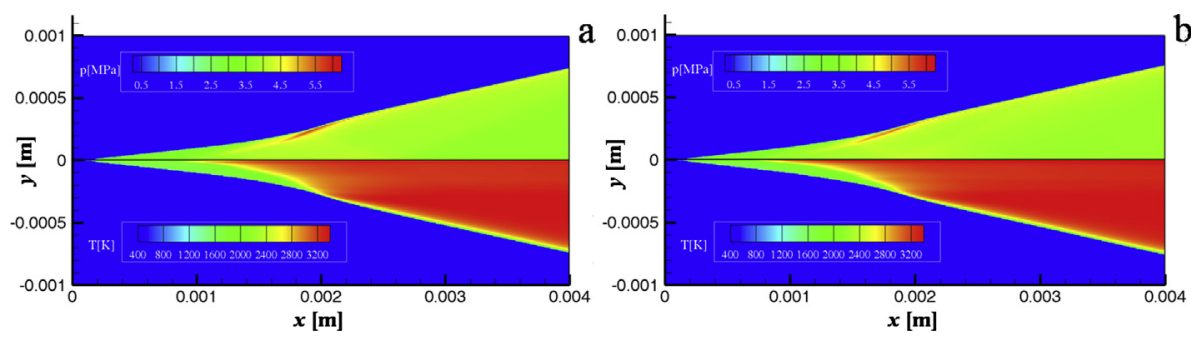

Fig. 3 - Pressure (upper) and temperature (lower) of inviscid (a) and viscous (b) ODW with $\mathbf{M}_{i}=10$.
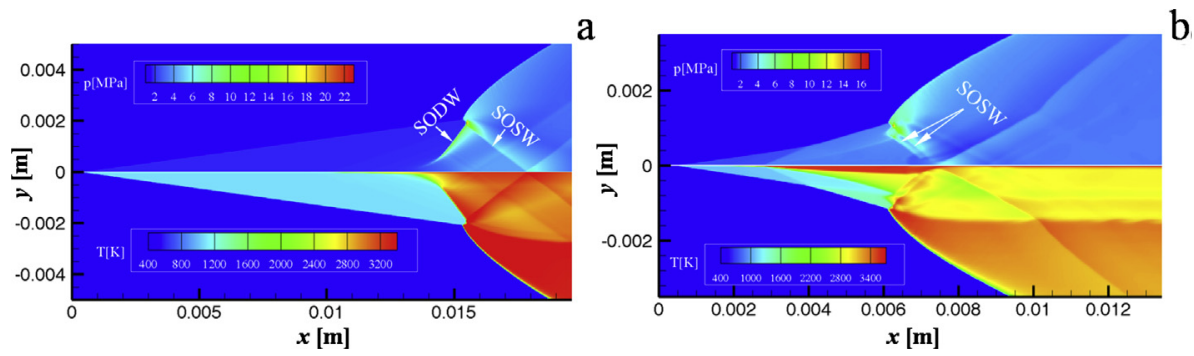

Fig. 4 - Pressure (upper) and temperature (lower) of inviscid (a) and viscous (b) ODW with $M_{i}=7$.

boundary layer, the high temperature zone near the wedge surface moves upstream, but the main structure does not change a lot. However, new structures with obvious differences from the inviscid one are generated in the case of $M_{i}=7$, as shown in Fig. 4b. It should be noted that to display the differences of ODW structures clearly, the corresponding plotted region has been adjusted at $M_{i}=7$. The transition type keeps the same, i.e. the abrupt transition, but the position of OSW-ODW moves to around $x=0.006 \mathrm{~m}$ from $0.015 \mathrm{~m}$ (in the inviscid flow field). A high temperature region appears near the wedge, suggesting that heat release occurs earlier. This high temperature region not only results in the forward movement of initiation region, but also influences the OSW surface. As illustrated in Fig. $4 \mathrm{~b}$, the OSW is not straight anymore, but composed of two sections. The section connected with the ODW has a larger oblique shock angle, which is attributed to the existence of the high temperature region near the wedge. Furthermore, the SODW disappears in the viscous ODW, but the SOSWs extending downstream still appear.

To verify whether the mesh used is enough to capture the ODW structures, different meshes are used and results are shown in Fig. 5. For both sets of meshes, the finest mesh with $0.005 \mathrm{~mm}$ in height is used in the inner part of the boundary layer (near the wedge surface), and the mesh height is amplified with a ratio of 1.05 along the vertical direction of the boundary layer. The mesh height of $0.005 \mathrm{~mm}$ corresponds a $\mathrm{Y}+$ value (dimensionless wall distance) of 30 . The coarse mesh uses an outer grid size of $0.050 \mathrm{~mm}$, while the fine mesh uses an outer grid size of $0.025 \mathrm{~mm}$. It is observed that there are no obvious differences between these two structures in Fig. 5. With the fine grid, the transition point of OSW-ODW moves downstream a little (from $\mathrm{x}=6.1 \mathrm{~mm}$ to $\mathrm{x}=5.9 \mathrm{~mm}$ ), and the error is about $3 \%$. Therefore, the mesh $(0.005-0.025 \mathrm{~mm})$ used in present simulations is thought to be enough to perform the structure investigations qualitatively, and it is used in the later analysis in this study.
Aforementioned results demonstrate that viscous effects should not be ignored in the abrupt ODW transition, but it is still unclear how these differences in ODW structures form. Theoretically, the viscous effects introduce dissipation everywhere in the whole flow field, but the difference is not

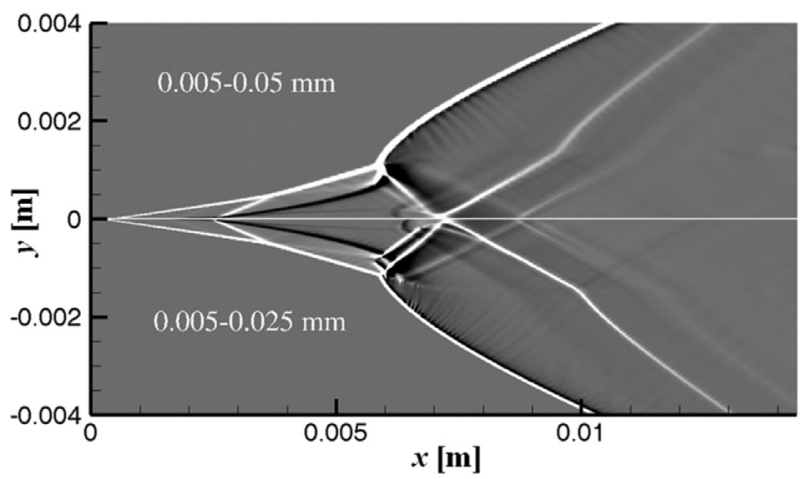

Fig. 5 - Numerical schlieren photos of viscous ODW with $M_{i}=7$ using different grids.

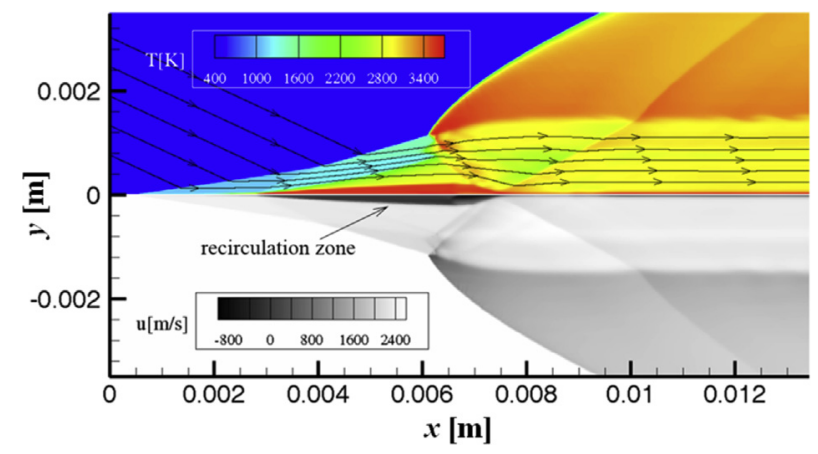

Fig. 6 - Contours of temperature (upper) and $x$-direction velocity (lower) in viscous ODW with $M_{i}=7$. 

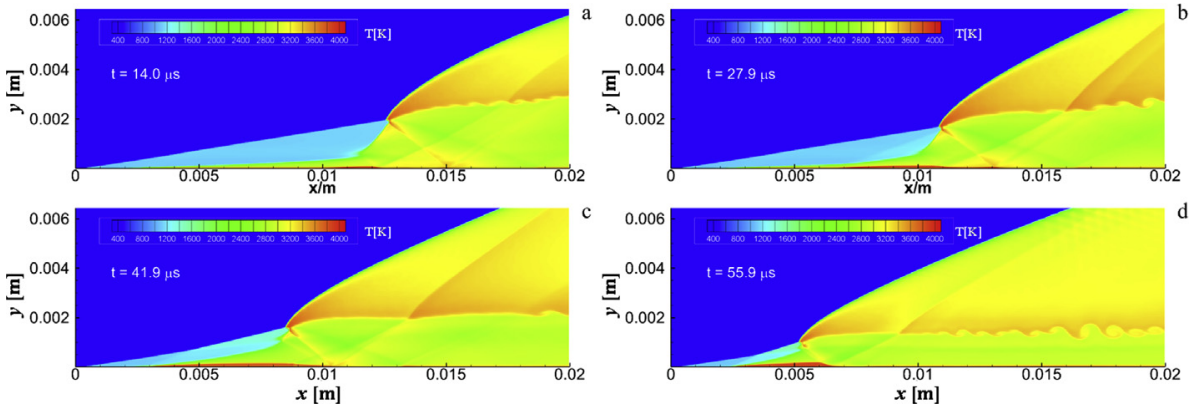

Fig. 7 - Evolution of viscous ODW with $M_{i}=7$.

obvious in the flow field behind the oblique detonation surface. Therefore, the viscous effects must be attributed into the formation of boundary layer. To further investigate the viscous ODW with $M_{i}=7$, its streamlines and contour of velocity component in the $x$-direction $(u)$ are displayed in Fig. 6. Obviously, the velocity $u$ is negative rather than positive in the high temperature region near the wedge, and the streamlines from upstream are raised there. The raised streamlines induce the two-section OSW, which has a larger oblique angle downstream. Therefore, it can be concluded that there is a recirculation region formed in the case of $M_{i}=7$, which changes the ODW structure. Due to the inclusion of boundary layer, it is expected that high temperature appears in the near wedge region. However, the boundary layer cannot change the structure so much, and the effect of recirculation dominates the main structure variation. Additionally, it is noteworthy that there are two SOSWs appearing in the initiation zone. This is mainly due to the existence of the recirculation region, which distorts the OSW surface. The pressure behind the downstream stronger OSW segment is higher than that in the region near the wall (from the upstream weaker OSW segment), leading to the formation of the first SOSW to balance the pressure variation. Higher pressure is also formed behind the main ODW, and consequently the second SOSW is formed to achieve pressure balance.

Numerical results above display the steady ODW structures. In this study, unsteady $\mathrm{N}-\mathrm{S}$ equations are solved and the formation process of ODW can be observed, which is helpful to understand the structure evolution. In inviscid ODW evolution process, the heat release starts downstream first. Then, the oblique angles of shock and detonation increase, and the initiation region moves upstream until the angles and initiation position reach their equilibrium values. Since this process has been studied before [37,38], it is not shown here. However, the structure evolution becomes different with the boundary layer taken into account, as shown in Fig. 7. The formation of inviscid ODW shown in Fig. 4 a takes about $10 \mu \mathrm{s}$, and similar process occurs in the viscous ODW formation. Thereafter, the ODW moves upstream with a relatively low speed, and it can be seen that at the instant of $14 \mu \mathrm{s}$, the initiation region moves from $x=0.015 \mathrm{~m}-0.012 \mathrm{~m}$. The viscous ODW reaches its equilibrium position at about $56 \mu \mathrm{s}$, after that the structure does not change further. In this process, the recirculation region grows gradually, but in Fig. 7a, it cannot be observed. Hence, the formation of recirculation region should be derived from the interaction of shock and boundary layer, which has also been observed before [39-41]. For smooth transition, there is no SOSW to be interacted with the boundary layer, and hence the thickness of the boundary layer within the ODW layer keeps thin, so it would not affect the ODW structure a lot.

\section{Conclusion}

In this study, the unsteady Navier-Stokes Equations in combination with a detailed chemical reaction model are used to simulate viscous ODWs in hydrogen-air mixtures. The effects of viscosity and hence of the boundary layer are focused by analyzing two typical ODW structures depending on $M_{i}$. The two basic structures have been studied before, and reproduced in this study. After including the viscosity, one structure keeps the same, while the other becomes different obviously. In the case of $M_{i}=10$ with smooth transition pattern of ODW, the structure keeps almost the same with the inclusion of viscosity. Nevertheless, in the case of $M_{i}=7$ with abrupt transition pattern of ODW, the inclusion of viscosity changes the structure obviously. The position of OSW-ODW moves to around $x=0.006 \mathrm{~m}$ from $0.015 \mathrm{~m}$, and the straight OSW becomes two sections, with the downstream one having a larger oblique angle. Moreover, further analysis founds that these differences are derived from the formation of recirculation zone within the ODW layer. For the abrupt transition in the case of $M_{i}=7$, there exists one oblique shock in the combustion product, which interacts with the boundary layer. The structure evolution of viscous ODW structures is examined as well, demonstrating how the recirculation zone forms in a relatively slow process. It should be noted that numerical viscosity is sometimes important to numerical simulations $[42,43]$. From the comparisons of the inviscid and viscous ODW results in present study, the same degree of numerical viscosity is involved in both cases, and the differences in structures of the viscous ODW from that of the inviscid ODW are unquestionably attributed to the inclusion of physical viscosity and hence the boundary layer.

Quantitative predictions of the initiation structures of ODW, especially the transition position of OSW to ODW, are rather important to design of the ODE combustor. Longer initiation zone may result in low fraction of oblique detonation combustion or even failure of ODW initiation, and hence 
the low propulsion performance or even failure of ODE. Therefore, this study emphasizes the great importance of considering viscosity in ODW simulations and future designs of ODE combustor.

\section{Acknowledgment}

This work was supported by the National Natural Science Foundation of China under Grant Nos. 11672308 and 11532014.

\section{REFEREN C ES}

[1] Fickett W, Davis WC. Detonation: theory and experiment. Dover Publications, Courier Corporation; 2000.

[2] Lee JHS. The detonation phenomenon. New York: Cambridge University Press; 2008.

[3] Lin L, Weng C, Chen Q, Jiao H. Study on the effects of ionization seeds on pulse detonation characteristics. Aero Sci Technol 2017;71:128-35.

[4] Wang Y, Le J. A hollow combustor that intensifies rotating detonation. Aero Sci Technol 2019;85:113-24.

[5] Sun J, Zhou J, Liu S, Lin Z, Lin W. Plume flowfield and propulsive performance analysis of a rotating detonation engine. Aero Sci Technol 2018;81:383-93.

[6] Lu FK, Fan H, Wilson DR. Detonation waves induced by a confined wedge. Aero Sci Technol 2006;10(8):679-85.

[7] Pratt DT, Humphrey JW, Glenn DE. Morphology of standing oblique detonation waves. J Propuls Power 1991;7(5):837-45.

[8] Li C, Kailasanath K, Oran ES. Detonation structures behind oblique shocks. Phys Fluids 1994;6(4):1600-11.

[9] Viguier C, Figueira da Silva LF, Desbordes D, Deshaies B. Onset of oblique detonation waves: comparison between experimental and numerical results for hydrogen-air mixtures. Symp (Int) Combust 1996;26(2):3023-31.

[10] Figueira da Silva LF, Deshaies B. Stabilization of an oblique detonation wave by a wedge: a parametric numerical study. Combust Flame 2000;121(1-2):152-66.

[11] Yang P, Teng H, Jiang Z, Ng HD. Effects of inflow Mach number on oblique detonation initiation with a two-step induction-reaction kinetic model. Combust Flame 2018;193:246-56.

[12] Yang P, Teng H, Ng HD, Jiang Z. A numerical study on the instability of oblique detonation waves with a two-step induction-reaction kinetic model. Proc Combust Inst 2019;37(3):3537-44.

[13] Zhang Y, Yang P, Teng H, Ng HD, Wen C. Transition between different initiation structures of wedge-induced oblique detonations. AIAA J 2018;56(10):4016-23.

[14] Teng H, Ng HD, Jiang Z. Initiation characteristics of wedgeinduced oblique detonation waves in a stoichiometric hydrogen-air mixture. Proc Combust Inst 2017;36(2):2735-42.

[15] Teng HH, Jiang ZL. On the transition pattern of the oblique detonation structure. J Fluid Mech 2012;713:659-69.

[16] Choi JY, Kim DW, Jeung IS, Ma F, Yang V. Cell-like structure of unstable oblique detonation wave from high-resolution numerical simulation. Proc Combust Inst 2007;31(2):2473-80.

[17] Teng HH, Jiang ZL, Ng HD. Numerical study on unstable surfaces of oblique detonations. J Fluid Mech 2014;744:111-28.

[18] Gui MY, Fan BC, Dong G. Periodic oscillation and fine structure of wedge-induced oblique detonation waves. Acta Mech Sin 2011;27(6):922-8.
[19] Verreault J, Higgins AJ, Stowe RA. Formation of transverse waves in oblique detonations. Proc Combust Inst 2013;34(2):1913-20.

[20] Teng H, Ng HD, Li K, Luo C, Jiang Z. Evolution of cellular structures on oblique detonation surfaces. Combust Flame 2015;162:470-7.

[21] Yang P, Ng HD, Teng H, Jiang Z. Initiation structure of oblique detonation waves behind conical shocks. Phys Fluids 2017;29(8). 086104.

[22] Zhang Y, Zhou L, Gong J, Ng HD, Teng H. Effects of activation energy on the instability of oblique detonation surfaces with a one-step chemistry model. Phys Fluids 2018;30(10). 106110.

[23] Liu Y, Wang L, Xiao B, Yan Z, Wang C. Hysteresis phenomenon of the oblique detonation wave. Combust Flame 2018;192:170-9.

[24] Liu Y, Wu D, Yao S, Wang J. Analytical and numerical investigations of wedge-induced oblique detonation waves at low inflow Mach number. Combust Sci Technol 2015;187(6):843-56.

[25] Gamezo VN, Khokhlov AM, Oran ES. The influence of shock bifurcations on shock-flame interactions and DDT. Combust Flame 2001;126(4):1810-26.

[26] Gamezo VN, Oran ES, Khokhlov AM. Three-dimensional reactive shock bifurcations. Proc Combust Inst 2005;30(2):1841-7.

[27] Choi JY, Jeung IS, Yoon Y. Scaling effect of the combustion induced by shock-wave boundary-layer interaction in premixed gas. Symp (Int) Combust 1998;27(2):2181-8.

[28] Liu Y, Han X, Lin Z, Zhou J. Numerical study on interaction of ramp-induced oblique detonation wave with a boundary layer, international conference on parallel computing in fluid dynamics. Berlin, Heidelberg: Springer; 2013. p. 504-13.

[29] Li C, Kailasanath K, Oran E. Effects of boundary layers on oblique-detonation structures. $31^{\text {st }}$ Aerospace Sciences Meeting 1993:450.

[30] Fusina G. Numerical investigation of oblique detonation wave for a shcramjet combustor. Ph.D. thesis. University of Toronto; 2003.

[31] Hirschel EH. Basics of aerothermodynamics. Springer-Verlag Berlin Heidelberg; 2005.

[32] Kim KH, Kim C, Rho OH. Methods for the accurate computations of hypersonic flows: I. AUSMPW+ scheme. J Comput Phys 2001;174(1):38-80.

[33] Burke MP, Chaos M, Ju Y, Dryer FL, Klippenstein SL. Comprehensive H2/O2 kinetic model for high-pressure combustion. Int J Chem Kinet 2012;44(7):444-74.

[34] Li J, Zhao Z, Kazakov A, Dryer FL. An updated comprehensive kinetic model of hydrogen combustion. Int J Chem Kinet 2004;36(10):566-75.

[35] Taylor BD, Kessle DA, Gamezo VN, Oran ES. Numerical simulations of hydrogen detonations with detailed chemical kinetics. Proc Combust Inst 2013;34(2):2009-16.

[36] McBride BJ, Zehe MJ, Gordon S. NASA Glenn coefficients for calculating thermodynamic properties of individual species. NASA/TP; 2002. Report No. 2002-211556.

[37] Teng HH, Zhao W, Jiang ZL. A novel oblique detonation structure and its stability. Chin Phys Lett 2007;24(7):1985.

[38] Teng H, Zhang Y, Jiang Z. Numerical investigation on the induction zone structure of the oblique detonation waves. Comput Fluids 2014;95:127-31.

[39] MacCormack RW. Numerical solution of the interaction of a shock wave with a laminar boundary layer, Proceedings of the second international conference on numerical methods in fluid dynamics. Berlin, Heidelberg: Springer; 1971. p. 151-63.

[40] Selig MS, Andreopoulos J, Muck KC, Dussauge JP, Smits AJ. Turbulence structure in a shock wave/turbulent boundarylayer interaction. AIAA J 1989;27(7):862-9. 
[41] Cai X, Liang J, Deiterding R, Mahmoudi Y, Sun M. Experimental and numerical investigations on propagating modes of detonations: detonation wave/boundary layer interaction. Combust Flame 2018;190:201-15.

[42] Cai X, Deiterding R, Liang J, Mahmoudi Y. Adaptive simulations of viscous detonations initiated by a hot jet using a high-order hybrid WENO-CD scheme. Proc Combust Inst 2017;36:2725-33.

[43] Cai X, Deiterding R, Liang J, Sun M, Mahmoudi Y. Diffusion and mixing effects in hot jet initiation and propagation of hydrogen detonations. J Fluid Mech 2018;836:324-51. 